\title{
PARTITIONING OF CLAY COLLOIDS AT AIR-WATER INTERFACES
}

\author{
Jiamin Wan and Tetsu K. Tokunaga
}

Earth Sciences Division, Lawrence Berkeley National Laboratory

Correspondence: Jiamin Wan

Address: Lawrence Berkeley National Laboratory,

One Cyclotron Road, MS 70-108B, Berkeley, California 94720

Telephone: 510-486-6004

Fax: 510-486-7797

Email: jwan@lbl.gov 


\begin{abstract}
Colloid sorption onto air-water interfaces in a variety of natural environments has been previously recognized, but better quantification and understanding is still needed. Affinities of clay colloids for the air-water interface were measured using a bubble-column method and reported as partition coefficients $(K)$. Four types of dilute clay suspensions were measured in $\mathrm{NaCl}$ solutions under varying $\mathrm{pH}$ and ionic strength conditions: kaolinite KGa-1, illite IMt-2, montmorillonite SWy-2, and bentonite. The $K$ values of three types of polystyrene latex particles with different surface-charge properties were also measured for comparison. Kaolinite exhibited extremely high affinity to the air-water interface at $\mathrm{pH}$ values below 7 . Illite has lower affinity to air-water interfaces than kaolinite, but has similar pH-dependence. Na-montmorillonite and bentonite clay were found excluded from the air-water interface at any given $\mathrm{pH}$ and ionic strength. Positively and negatively charged latex particles exhibited sorption and exclusion, respectively, at the air-water interface. These results show the importance of electrostatic interactions between the air-water interface and colloids, especially the influence of $\mathrm{pH}$ dependent edge charges, and influence of particle shape.
\end{abstract}

Key Words: colloids, air-water interface, partitioning coefficient, bubble-column, kaolinite, montmorillonite, illite, latex microspheres, $\mathrm{pH}$-dependent charges. 


\section{INTRODUCTION}

Colloids have been found favorably sorbed at air-water interfaces in many natural environments. For instance, enrichment of colloid-associated heavy metals and organic compounds at the surface of oceans and lakes have been reported (1-4). Clays, humic acids, and microorganisms have also been found preferentially sorbed at air-water interfaces in unsaturated soils (5-9). Colloid partitioning at gas-water interfaces has been analyzed as a special case of heterocoagulation, the interaction between double-layers of dissimilar surfaces $(10,11)$. Unlike partitioning of larger-sized particles, electrostatic partitioning of hydrophilic colloids at air-water interfaces can occur without deformation of the air-water interface and without capillary effects $(11,12)$. Predictions from heterocoagulation analysis of colloid flotation include the dominance of interfacial forces over inertial forces, and the requirement of either low-magnitude zeta potentials or zeta potentials of opposite signs for the colloids and air-water interface. In the absence of surfactants, zeta potentials for the air-water interface are negative $(13,14)$. The $\mathrm{pH}-$ dependent negative charge at the air-water interface is attributed to hydrogen bonding of hydroxyl ions to interfacial water molecules $(15,16)$. Thus, the dominant influence of the repulsive electrostatic force prevents partitioning of negatively charged colloids at air-water interfaces, except when surface potentials are low and ionic strength is high (11).

In subsurface environments, gas-water interfaces exist discontinuously below the water table (trapped gas) and much more continuously above the water table in the vadose zone. In groundwaters, colloids attached on gas bubbles can move with the bubbles. In the vadose zone, colloids at air-water interfaces may be mobile or immobile $(7,8,17)$. The air-water interfacial area can be quite large in vadose zone soils and sediments. Since clay minerals are ubiquitous, abundant, and high in sorptivity for contaminants, quantification of their partitioning at air-water 
interfaces may be important in understanding some vadose zone geochemical transport processes.

For solutions containing surface-active molecules, measurement of changes in surface tension resulting from changes in solute concentration permits calculation of their surface excess through the Gibbs adsorption equation. However, this approach is typically insensitive to suspensions of particles, i.e., surface tension changes resulting from colloid partitioning are not measurable under most environmentally relevant conditions. Thus, several alternative approaches to characterizing surface accumulations of particles and associated species have been developed. Previously developed methods include surface microlayer sampling using stainlesssteel mesh, skimmer devices, slides, and jet drop collection $(4,18,19)$. These methods can have large uncertainties in thicknesses and sampled interfacial areas, and results are often reported as enrichment factors for a given estimated thickness. In studies of surfactant-enhanced fineparticle flotation, colloid partitioning has been characterized in terms of flotation-rate constants (20). However, the experimental methods used in such studies require surfactants to rapidly remove captured particles from the foam phase. To quantify colloid partitioning at air-water interfaces under surfactant-poor conditions typical of subsurface environments, an alternative method is needed. Recently, Wan and Tokunaga (21) developed a simple dynamic method to characterize colloid surface excesses at air-water interfaces without requiring assumptions about the thickness of interfacial regions and without use of surfactants. In this paper we report measured partition coefficients of different clay minerals and latex microspheres at the air-water interface, primarily under environmentally relevant solution-chemistry conditions. For this purpose, no surfactants were used, and most experiments were conducted at low values of ionic strength. 


\section{METHODS AND MATERIALS}

\section{Bubble-Column Method and Definition of Colloid Partition Coefficient}

Wan and Tokunaga (17) developed a bubble-column method to measure surface excesses of colloids at the air-water interface, based on a design by Lemlich $(22,23)$. Using this method, air is bubbled through a vertical column containing a dilute aqueous suspension of colloids, with a free surface at the top. Rising bubbles sorb and carry surface-active species upwards, then release them back to the solution at the free surface where the bubbles burst. Upon reaching steady state, the concentration profile along the column length reflects the balance between upward transport (by partitioning onto rising bubbles) and downward transport (by eddy dispersion). This balance is characterized locally by

$$
a f \Gamma=A D \frac{d C}{d z}
$$

where $a$ is the surface area per bubble, $f$ is the bubble generation rate $\left[\mathrm{T}^{-1}\right], \Gamma$ is the surface

excess $\left[\mathrm{L}^{-2}\right], A$ is the column cross-sectional area, $D$ is the column eddy dispersion coefficient $\left[\mathrm{L}^{2}\right.$ $\left.\mathrm{T}^{-1}\right], C$ is the local colloid concentration in suspension $\left[\mathrm{L}^{-3}\right]$, and $z$ is the vertical coordinate (positive upwards). For dilute suspensions, partitioning of colloids at the water-gas interface is assumed to be linearly dependent on the local suspended colloid concentration, such that

$$
\Gamma=K C
$$

If colloid partitioning is sufficiently rapid such that Eq. [2] represents local equilibria, then $K$ is an equilibrium partition coefficient [L]. Equilibrium $K$ values were obtained for surfactant 
partitioning in bubble columns (21), as verified through comparisons with surface tension measurements. However, lacking independent equilibrium measurements of colloid partitioning at air-water interfaces, we must regard $K$ values obtained for colloids as being less than or equal to their equilibrium values. The solution to Eq. [1] and [2] is an exponential profile, and the steady-state ratio of the concentration at elevation $\mathrm{z}$ versus the bottom of the column is

$$
\frac{C(z)}{C_{b}}=\exp (J z)
$$

where

$$
J=\frac{a f K}{A D}
$$

The initial (uniform) concentration $\left(\mathrm{C}_{\mathrm{o}}\right)$ and the steady-state concentration at the bottom of the column $\left(\mathrm{C}_{\mathrm{b}}\right)$ are related through Eq. [2] and mass conservation, giving

$$
C_{b}=\left\lfloor\frac{H J}{\exp (H J)-1}\right\rfloor C_{o}
$$

such that

$$
\frac{C(z)}{C_{O}}=\left\lfloor\frac{H J}{\exp (H J)-1}\right\rfloor \exp (J z)
$$

Thus, when $A, a, f$, and $D$ have been independently determined, measurement of steady-state $C(z)$ permits a unique determination of $K$.

\section{Experimental Conditions and Parameters Used in the Model Calculations}

The bubble column described in Wan and Tokunaga (21) was also used in this study. The glass column was $2.0 \mathrm{~m}$ in height and had a $0.019 \mathrm{~m}$ inner diameter, with eight septum- 
capped sampling ports along the length of the column. Air bubbles are generated by forcing filtered compressed air into the chamber located at the bottom of the column, and up through a fritted glass plate. Airflow was controlled and measured with a flow control system (ColeParmer), and a pressure transducer was used for monitoring the inflow pressure. An airflow rate of 10.0 standard $\mathrm{ml} \mathrm{min}^{-1}$ was used (as it was in all the experiments reported in this paper). This flow rate was chosen because it provided a large concentration of relatively uniformly sized spherical bubbles, without air channeling.

Bubble sizes were measured photographically using a CCD camera recording and computer image analyses. A $254 \mu \mathrm{m}$ diameter wire was installed as the scale inside of the column during photographing. Three recorded images under each chemical conditions were used to determine the average bubble diameter. All the bubbles, excluding the ones close to the glass walls that exhibited optical distortion, were measured in each image. Total surface area of the measured bubbles was calculated (assuming spherical shape), from which an area-averaged bubble diameter was determined. The difference between measured average bubble diameters in $1.0 \mathrm{mM}$ and $100 \mathrm{mM} \mathrm{NaCl}$ solutions (containing diluted kaolinite particles) was within the range of measurement error. An average bubble diameter of $481 \pm 71 \mu \mathrm{m}$ was used in all calculations; this diameter presents an effectively flat air-water interface to the much smaller $(\approx 1 \mu \mathrm{m})$ particles. The column eddy dispersivity, $D$, of $6.0 \times 10^{-4} \mathrm{~m}^{2} \mathrm{~s}^{-1}$ was experimentally determined through measuring dispersion of step inputs of a salt tracer in the bubble column. The time required to reach the steady-state was determined experimentally. A length of 2 to 3 hours was found optimum, and longer times resulted in aggregation of colloids under some tested conditions. 
All experiments were conducted at room temperature $\left(22.8 \pm 0.5{ }^{\circ} \mathrm{C}\right)$. At steady-state, samples $(2 \mathrm{ml}$ each) were withdrawn through the septum ports using syringes, starting with the top port and progressively collecting from lower ports. The relative concentrations of the samples were measured with a spectrophotometer (Milton Roy 601, Rochester, NY) in the linear absorbance range. Samples were analyzed immediately after being withdrawn from the column and also after sonicating for 10 minutes to break down possible colloidal aggregates formed during bubbling. Absolute particle concentrations were determined with a particle sizer-counter (Multisizer II, Coulter Co., Miami, FL). The measured concentration profile (relative concentration versus the distance along the column) from each experiment was used with Equations 4 and 6 to obtain the partition coefficient, $K$, for the specific experimental conditions (colloid type and solution chemistry).

\section{Preparation of Colloid Suspensions}

Both clay colloids and polystyrene latex microspheres were measured for their partitioning coefficients at the air-water interface (Table 1). The clays tested were kaolinite (KGa1, well crystallized), Na-montmorillonite (SWy-2), and illite (IMt-2), all purchased from the Clay Minerals Society (Columbia, MO). SEM images of these clays are shown in Figure 1. Some tests were also done on a bentonite clay, separated from Wyoming bentonite rock (American Colloid Company, Arlington Heights, IL).

To prepare clay suspensions, a sample of $50 \mathrm{~g}$ clay was suspended in 1 liter of distilled water by vigorous stirring in a blender, adjusting the $\mathrm{pH}$ of the suspension to 9.0 by addition of

$0.01 \mathrm{M} \mathrm{NaOH}$. After overnight equilibration, the suspension was size-fractionated by centrifugation to collect particles in the equivalent size range of 0.6 to $1.0 \mu \mathrm{m}$ (equivalent- 
spherical-diameter). Particles greater than $1.0 \mu \mathrm{m}$ (equivalent sphere basis) were discarded in order to minimize the influence of sedimentation. Particles less than $0.6 \mu \mathrm{m}$ were also discarded because they were below the detection limit of our particle counter (Coulter Multisizer II, with $50 \mu \mathrm{m}$ orifice). The experimentally constrained choice of working with colloids in the 0.6 to 1.0 $\mu \mathrm{m}$ range is disadvantageous from considerations of particle-bubble collision efficiency since finer sizes permit more Brownian encounters while larger sizes permit inertial contact $(24,25)$. Minimum collision efficiencies for colloid encounters with interfaces occur in the 0.6 to $1.0 \mu \mathrm{m}$ size range.

It should also be noted that hydrodynamic friction-dependent size fractionation methods (e.g., sedimentation, centrifugation, electrophoresis) are particle shape-dependent. Clay platelets experience significantly more drag per unit mass as their aspect (plate diameter to thickness of aggregate particle) ratios depart from unity. Electron micrograph images of our kaolinites (e.g., Fig. 1a) appear to show aspect ratios varying within the range of 2 to 10 measured for KGa-1 by Brady et al. (26), and thicknesses in the range of 40 to $70 \mathrm{~nm}$. Within this range of aspect ratios, the friction factor for an equivalent oblate ellipsoid only increases by $4 \%$ to $46 \%$, respectively, relative to that of a sphere (27). Our images of illite and montmorillonite (Figs. 1b and 1c) indicate progressively higher aspect ratios. Reported values of illite platelet thicknesses tend to fall in the range of 8 to $20 \mathrm{~nm}(28$ - 30), while smectites dispersed in Na-suspensions are typically regarded as sheets of $1 \mathrm{~nm}$, unit cell thickness $(28,31)$. Quantification of the aspect ratios are difficult from SEM images, especially for montmorillonite because they are likely to involve stacking of many individual sheets that were previously dispersed in the dilute $\mathrm{NaCl}$ test solutions. 
After size fraction by centrifugation, particles were washed with a saline, acid solution $(1.0 \mathrm{mM} \mathrm{HCl}+1.0 \mathrm{M} \mathrm{NaCl})$. The desired size fraction was redispersed in 1 liter of saline acid solution and stirred overnight. Clay flocculates were washed with $1.0 \mathrm{mM} \mathrm{NaCl}$ until pH was raised to 5.5 (about 5 times by centrifugation). In the final step, the particles were redispersed in $1.0 \mathrm{mM} \mathrm{NaCl}$ to a concentration of about $10^{9}$ particles $\mathrm{mL}^{-1}$, and $\mathrm{pH}$ was adjusted to 8.5 . These stock clay suspensions were stored in a refrigerator $\left(4{ }^{\circ} \mathrm{C}\right)$ for later experiments. Dilute suspensions, about $5 \times 10^{6}$ particles $\mathrm{mL}^{-1}$, were used in the column experiments. The absolute particle concentration of a stock suspension was measured using a particle analyzer (Coulter Multisizer II). The desired final $\mathrm{pH}$ of a suspension was achieved by addition of $0.01 \mathrm{M} \mathrm{HCl}$ or $0.01 \mathrm{M} \mathrm{NaOH}$. $\mathrm{NaHCO}_{3}$ and/or $\mathrm{Na}_{2} \mathrm{CO}_{3}$ or $\mathrm{Na}_{2} \mathrm{HPO}_{4}$ was used to buffer suspensions for $\mathrm{pH}$ values greater than 7 .

Three types of surfactant-free polystyrene particles (Interfacial Dynamics Corp., Portland, OR) were tested for comparison with clays. Carboxyl polystyrene latex particles are negatively charged $\left(14\right.$ charge sites per $\left.\mathrm{nm}^{2}\right)$, and $1.0 \mu \mathrm{m}$ in diameter. Sulfate polystyrene latex particles are slightly negatively charged $\left(0.4\right.$ charge sites per $\left.\mathrm{nm}^{2}\right)$, and $1.0 \mu \mathrm{m}$ in diameter. The amidine polystyrene latex particles are positively charged $\left(0.9\right.$ charge sites per $\left.\mathrm{nm}^{2}\right)$, and 0.60 $\mu \mathrm{m}$ in diameter. Each set of particles was dispersed in $1.0 \mathrm{mM} \mathrm{NaCl}$, pH 5.7 solutions to achieve bubble column particle concentrations of about $10^{7}$ particles $\mathrm{mL}^{-1}$.

\section{RESULTS}

Surface excesses of kaolinite particles at air-water interfaces were measured using diluted suspensions in $1.0 \mathrm{mM} \mathrm{NaCl}$, at $\mathrm{pH}$ values ranging from 4 to 9 . The measured partition coefficients revealed high affinities of kaolinite to the air-water interface under acidic conditions. 
A set of profile samples taken at steady-state from a kaolinite column ( $\mathrm{pH} \mathrm{5.7)}$ is shown in Figure 2a. A distinct concentration gradient along the column was easily visible after only about 10 minutes. Kaolinite particles were highly concentrated at the top, and almost absent from the bottom of the column. The corresponding relative concentration profile of the samples shown in Fig. $2 \mathrm{a}$ is shown in Fig. $2 \mathrm{~b}$ (pH 5.7 profile). The relative concentrations $\mathrm{C}_{\mathrm{o}}$ varied from 5 at the top to near zero at the bottom, and the absolute particle numbers varied by three orders of magnitude. Fig. 2b also shows typical measured relative concentration profiles at $\mathrm{pH}$ values of 4.5, 6.3 and 7.5. The curves are least-squares fits to the data, using eq. 6. Larger concentration gradients reflect higher degree of sorption at the air-water interface, and therefore larger $K$ values. A total of 40 measurements were conducted under different $\mathrm{pH}$ conditions, with data summarized in Fig. 3. The measured $K$ values are in the range of 0 to $200 \mu \mathrm{m}$, depending on $\mathrm{pH}$. In the $\mathrm{pH}$ range of 4 to $6.3, K$ values vary within the range of experimental uncertainty. An average value of $165 \pm 24 \mu \mathrm{m}(14 \%$ uncertainty) was obtained. $K$ decreases rapidly when $\mathrm{pH}$ exceeds 6.3, and reaches zero as $\mathrm{pH}$ approaches $8 . K$ values also decrease when the $\mathrm{pH}$ is lower than 4. The ionic-strength dependence of $K$ values for kaolinite was studied in solutions of $10^{-1}$ to $10^{-5} \mathrm{M} \mathrm{NaCl}$ (Fig. 4). For this set of tests, the solution $\mathrm{pH}$ was maintained at 5.6 for all the experiments except the ones at $10^{-3} \mathrm{M}$, which had $\mathrm{pH}$ values ranging from 5.4 to 5.8. No distinct difference was detected in the ionic-strength range of $10^{-1}$ to $10^{-3} \mathrm{M}$. A slightly decreased $K$ was seen at an ionic-strength of $10^{-4} \mathrm{M}$, and a large decrease was measured when ionic-strength was dropped to $10^{-5} \mathrm{M}$.

The partitioning of illite clay at the air-water interface was measured in $1.0 \mathrm{mM} \mathrm{NaCl}$, with $\mathrm{pH}$ varying from 4 to 9 . Compared to kaolinite, illite is slightly surface active. Measured $K$ 
values are in the range of 0 to $30 \mu \mathrm{m}$ (Fig. 5), with an average $K$ value of $20 \pm 5 \mu \mathrm{m}$ at $\mathrm{pH}<6.5 . K$ decreases with increasing $\mathrm{pH}$ and reaches zero when $\mathrm{pH}$ approaches 9.

Measurements for Na-montmorillonite and bentonite $K$ were conducted in $1.0 \mathrm{mM} \mathrm{NaCl}$, over a $\mathrm{pH}$ range of 4 to 8 . These clays did not partition at the air-water interface under the tested conditions. The influence of ionic strength was tested in solutions of $10^{-1}$ to $10^{-5} \mathrm{M} \mathrm{NaCl}$ at $\mathrm{pH}$ 5.5, and found to have no measurable influence. Inverted concentration profiles were developed at all the $\mathrm{pH}$ and ionic strength conditions tested, with particle concentrations being higher at the bottom and lower at the top of the columns (Fig. 6). Surface exclusion of salts in solution is commonly measurable through increases in surface tension. Negative concentration gradients of simple salts at high concentrations were measured in bubble columns, as expected due to their surface exclusion (20). Like simple salt solutions, negative concentration gradients of colloids observed in this study are also an indication of surface exclusion. Although the bubble column method is not sensitive enough for quantifying surface exclusion, it can distinguish between surface exclusion and surface accumulation.

The polystyrene latex microspheres exhibited slightly negative to slightly positive partitioning at the air-water interface. Negatively charged carboxylated microspheres yielded $K$ values of $-3 \pm 4 \mu \mathrm{m}$. The $K$ for the nearly neutral sulfate microspheres was essentially zero ( $1 \pm 1$ $\mu \mathrm{m})$. The positively charged amidine microspheres showed slightly favorable partitioning at the air-water interface, with $K$ values ranging from 4 to $13 \mu \mathrm{m}$.

\section{DISCUSSION}

The results from bubble column measurements of colloid partitioning at the air-water interface collectively reflect the importance of particle charge, solution ionic strength, and 
particle geometry (including distribution of charge sites). Since we are considering the variety of colloid affinities with air-water interfaces, it is worth beginning with reviewing charge characteristics of the air-water interface. Zeta $(\zeta)$ potential measurements of surfactant-free airwater interfaces yield negative values. In deionized water, the air-water interfacial $\zeta=-65 \mathrm{mV}$ (14). The solution $\mathrm{pH}$ associated with that value was not reported, but it corresponds to results obtained by $\mathrm{Li}$ and Somasundaran (13) in dilute $\left(10^{-5} \mathrm{M}\right) \mathrm{NaCl}$ at a $\mathrm{pH}$ of about 5.5. They reported air-water interface $\zeta$ potential measurements over a very wide $\mathrm{pH}$ range (2 to 12 ) for $\mathrm{NaCl}$ concentrations up to $0.1 \mathrm{M}$. The $\zeta$ potentials of air bubbles in these $\mathrm{NaCl}$ solutions were all negative (ranging from -10 to $-90 \mathrm{mV}$ ), decreased with increasing $\mathrm{pH}$, and increased with increasing ionic strength. They also reported slightly positive values of $\zeta$ at $\mathrm{pH}>9$ when $\mathrm{Mg}$ was added at concentrations high enough to precipitate $\mathrm{Mg}(\mathrm{OH})_{2}$ particles on bubble surfaces. These previous studies indicate that the air-water interface is effectively negatively charged under all of our test conditions. It is also worth noting that very similar $\zeta(\mathrm{pH})$ values were obtained for oilwater interfaces by Marinova et al. (15).

The influence of the surface charge of a colloid on its partitioning can now be considered in light of the aforementioned information on the air-water interfacial charge. The experiments on polystyrene latex microspheres provide the clearest test of charge effects since these particles are all spherical, each having a uniform distribution of a specific charge group, and all were tested in dilute $\mathrm{NaCl}$ solutions. Only the positively charged amidine microspheres exhibited partitioning at the air-water interface $(K \approx 7 \mu \mathrm{m})$, while the negatively charged carboxylate and sulfate microspheres showed insignificant partitioning. These results are consistent with electrostatic attraction of positively charged particles to the negatively charged air-water interface. 
The dominant influence of electrostatic interactions between colloids and the air-water interface is further evident from $K$ values of mineral colloids, especially from measurements of $\mathrm{pH}$ and ionic strength dependence. Kaolinite KGa-1 may be the least complicated of our mineral colloids to analyze because of its small permanent charge, and well characterized $\mathrm{pH}$-dependent charge and geometry $(26,32,33)$. It has a small permanent negative charge largely on its siloxane surface associated with $\mathrm{Al}(\mathrm{III})$ substitution, and $\mathrm{pH}$ dependent charge arising from protonating-deprotonating aluminol and silanol edge sites. Based on the observed behavior of latex microspheres, negatively charged face surfaces of kaolinite are not expected to partition at the air-water interface under the conditions tested, while its protonated edge sites can sorb at this interface. Kaolinite begins to partition at air-water interfaces as the $\mathrm{pH}$ is lowered below 7.5, and reaches its highest $K$ values (100 to $200 \mu \mathrm{m}$ ) under acidic $\mathrm{pH}$. This behavior parallels measurements of the $\mathrm{pH}$-dependent anion adsorption envelopes of kaolinites, which diminish to zero for $\mathrm{pH}>7$ (34). The $\mathrm{pH}$ at which kaolinite $\mathrm{KGa}-1$ is at its point of zero net proton charge (PZNPC) is about $5(32,35)$. The fact that measurable $K$ values were obtained for kaolinite even at $\mathrm{pH}$ values substantially higher than the PZNPC indicates that it can partition at air water interfaces even when only a small fraction of edge sites are protonated. Over the tested $\mathrm{pH}$ range and the short testing times, we believe that kaolinite dissolution was minor even in the acidic solutions, and that the primary change imposed on kaolinite particles in the course of decreasing $\mathrm{pH}$ is protonation of its edge sites. Despite its small permanent negative charge, the attraction of positively charged edges to the air-water interface permits partitioning of kaolinite.

Such partitioning requires not only sufficient edge charge density, but sufficient edge surface area as well. Among the common aluminosilicate clays, kaolinites appear to be particularly suited for edge surface partitioning at air-water interfaces because its edges 
contribute 20 to $30 \%$ of its total surface area $(26,33)$. Furthermore, kaolinite particles tend to occur as hexagonal plates with well-defined, planar edge surfaces (Fig. 1a) that maximize interactions with effectively planar air-water interfaces. The double layer influence on efficiency of kaolinite edge encounters with bubble surfaces is reflected in the ionic strength dependence of partitioning (Fig. 4). At low ionic strength, greater double layer thicknesses diminish particleinterface encounters. The extent to which kinetic limitations may contribute to underestimating $K$ values has not been determined.

Partitioning of illite at the air-water interface followed $\mathrm{pH}$-dependent trends similar to that obtained for kaolinite, but with smaller K. Differences between these two types of colloids include their layer structure, intrinsic charge, and overall shape. Although the 2:1 illite is composed of an aluminum octahedral layer between two silica tetrahedral layers, whereas kaolinite is a 1:1 aluminosilicate, the $\mathrm{pH}$ dependence of edge charges are very similar $(31,29)$. However, illites have higher permanent negative charge, higher aspect ratios, and less planar edges (Fig. 1b) relative to kaolinite. These characteristics all hinder sorption of illite at air-water interfaces. Thus, the pH-dependent partitioning of illite at air-water interfaces is expected to be similar to that of kaolinite, but of weaker magnitude.

The lack of montmorillonite and bentonite partitioning at air-water interfaces under any of the tested conditions can be interpreted in terms of the same criteria used for kaolinite and illite: edge charge, intrinsic charge, and particle shape. Since montmorillonite and bentonite are 2:1 aluminosilicate clays, they have $\mathrm{pH}$-dependent edge charges similar to that of illite that favor partition at the air-water interface under neutral to acidic conditions. However the much higher permanent negative charges of montmorillonite and bentonite provide a substantial repulsive interaction with air-water interfaces. These smectite clays also become fully dispersed in dilute 
$\mathrm{Na}$ suspensions, resulting in very high aspect ratios exceeding $10^{2}$. Such high aspect ratios, combined with more irregular frayed edges (Fig. 1c) permit very limited edge surface contact with the effectively planar air-water interface. Furthermore, at high aspect ratios (36) and more specifically, very thin single sheets of $2: 1$ clays $(37,38)$, the negative electrostatic field emanating from smectite face surfaces overwhelms the positive edge field. These considerations collectively indicate that fully dispersed Na-smectites will not partition at air-water interfaces, and are consistent with the bubble column measurements.

It should be noted that the results presented were all from colloid suspensions in $\mathrm{NaCl}$ solutions. The influence of solution chemistry, in particular cation valence, is expected to be important as well. Smectite suspensions in Ca solutions may be less dispersed, hence consist of stacked particles with lower overall aspect ratios and potentially larger influences of edge charges. The possibility of smectite partitioning at air-water interfaces through cation bridging also arises in divalent solutions.

\section{CONCLUSIONS}

Using the bubble column method, we measured partitioning of common aluminosilicate clays and latex microspheres at surfactant-free air-water interfaces in $\mathrm{NaCl}$ solutions. Comparisons among microspheres showed that only positively charged particles partitioned at the air-water interface. Kaolinite and illite exhibited pH-dependent partitioning to the air-water interface, diminishing above $\mathrm{pH} \approx 8$. The very large partitioning of kaolinite was attributed to its large fraction of flat edge surface area that is progressively protonated with lower $\mathrm{pH}$, and its lower intrinsic negative charge density. By extension, colloidal oxide particles such as goethite and gibbsite are expected to have very high, $\mathrm{pH}$-dependent affinities to the air-water interface. 
The absence of montmorillonite and bentonite partitioning at the air-water interface is believed to result from the high permanent negative charge, very low edge surface area, and spill-over of the negative electric field from their face surfaces over their very thin edges. The nature of the ionic solution (e.g., $\mathrm{NaCl}$ vs. $\mathrm{CaCl}_{2}$ or $\mathrm{CaSO}_{4}$ ) is also expected to influence clay colloid partitioning at air-water interfaces through aggregation and cation bridging.

Although the vast majority of clays in soils are part of the immobile solid phase matrix, there is interest in colloids suspended in the mobile aqueous phase because they transport contaminants. Since the air-water interfacial area can be large in unsaturated soils, it is important to understand the distribution of colloids between this interface and the bulk soil solution. The measurements presented here provide the first contributions to a database of partition coefficients for environmental colloids at the air-water interface.

\section{ACKNOWLEDGMENTS}

This work was carried out under U.S. Department of Energy Contract DE-AC03-76SF-00098. Funding was provided by the U.S. Department of Energy, Basic Energy Science, Geosciences

Research Program. We appreciate the technical support provided by Eduardo Saiz, Richard Stover, and Keith Olson of LBNL.

\section{REFERENCES}

1. Sutcliffe, W. H., Baylor, Jr., E. R., and Menzel, D. W., Deep Sea Res. 10, 233 (1963).

2. Duce, R. A., Quinn, J. G., Olney, C. E., Piotrowicz, S. R., Ray, B. J., Wade, T. L., Science 14, 161-163 (1972).

3. MacIntyre, F., Sci. Am. 230, 62 (1974). 
4. Gershey, R. M., Limnol. Oceanog. 28, 309-319 (1983).

5. Chen, Y., Schnitzer, M. Soil Sci. 7-15, 125 (1972).

6. Goldenberg, L. C., Hutcheon, I., Wardlaw, N., Transport Porous Media 4, 129 (1989).

7. Wan, J., and Wilson, J. L., Water Resour. Res. 30, 11 (1994).

8. Wan, J., Wilson, J. L., and Kieft, T. L., Appl. Environ. Microbiol. 60, 509 (1994).

9. Powelson, D. K., and Mills, A. L., Appl. Environ. Microbiol. 62, 2593 (1996).

10. Derjaguin, B. V., and Dukhin, S. S., Trans. Inst. Min. Metall. 70, 221 (1960).

11. Derjaguin, B. V., Dukhin, S. S., Rulyov, N. N., Surf. Colloid Sci. 13, 71 (1984).

12. Earnshaw, J. C., J. Phys. D. Appl. Phys. 19, 1863 (1986).

13. Li, C., and Somasundaran, P., J. Colloid Interface Sci. 146, 215 (1991).

14. Graciaa, A., Moel, G., Saulner, P., Lachaise, J., and Schechter, R. S., J. Colloid Interface Sci. 172, 131 (1995).

15. Marinova, K. G., Alargova, R. G., Denkov, N. D., Velev, O. D., Petsev, D. N., Ivanov, I. B., and Borwankar, R. P., Langmuir 12, 2045 (1996).

16. Kralchevsky, P. A., Danov, K. D., and Denkov, N. D., "Chemical physics of colloid systems and interfaces", Chapter 11, p. 333-494, in Birdi, K. S., Handbook of Surface and Colloid Chemistry, CRC Press, Boca Raton, 1997.

17. Wan, J.; Tokunaga, T.K.. Environ. Sci. Technol. 31, 2413-2420 (1997).

18. Blanchard, D. C., and Syzdek, L., Science 170, 626 (1970).

19. Schafer, A., Harms, H., and Zehnder, A. J. B., Environ. Sci. Technol. 32, 3704 (1998).

20. Collins, G. L., and Jameson, G. J., Chem. Eng. Sci. 31, 985 (1976).

21. Wan, J., and T. K. Tokunaga, Environ. Sci. Technol. 32, 3293 (1998).

22. Lemlich, R. AIChE J 12, 802 (1966). 
23. Lemlich, R. in Recent Developments is Separation Science: Li, N. N., editor, Chemical Rubber Company, Cleveland, OH, 1972.

24. Reay, D., and Ratcliff, G. A., Can. J. Chem. Eng. 51, 178 (1973).

25. Reay, D., and Ratcliff, G. A., Can. J. Chem. Eng. 53, 481 (1975).

26. Brady, P. V., Cygan, R. T., and Nagy, K. L., J. Colloid Interface Sci. 183, 356 (1996).

27. Hiemenz, P. C., Principles of Colloid and Surface Chemistry, $2^{\text {nd }}$ edition, p. 815, Marcel Dekker, Inc., New York, 1986.

28. Nadeau, P. H., Clay Minerals 20, 499 (1985).

29. Beene, G. M., Bryant, R., and Williams, D. J. A., J. Colloid Interface Sci. 147, 358 (1991).

30. Drits, V. A., Eberl, D. D., and Srodon, J., Clays Clay Min. 46, 38 (1998).

31. Van Olphen, H., An Introduction to Clay Colloid Chemistry, 2nd Ed., John Wiley and Sons, New York, 1977.

32. Schroth, B. K., and Sposito, G., Clays Clay Minerals 45, 85 (1997).

33. Zbik, M., and Smart, R. St.C., Clays Clay Mineral 46, 153 (1998).

34. Ferris, A. P., and Jepson, W. B., J. Colloid Interface Sci. 51, 245 (1975).

35. Sposito, G., Environ. Sci. Technol. 32, 2815 (1998).

36. Secor, R. B., and Radke, C. J., J. Colloid Interface Sci. 103, 237 (1985).

37. Chang, F.-R. C., and Sposito, G., J. Colloid Interface Sci. 163, 19 (1994).

38. Chang, F.-R. C., and Sposito, G., J. Colloid Interface Sci. 178, 555 (1996). 


\begin{tabular}{|l|r|r|l|l|}
\hline colloid & $\begin{array}{l}\text { total specific } \\
\text { surface area }\end{array}$ & $\begin{array}{l}\text { structural } \\
\text { charge }\end{array}$ & $\begin{array}{l}\text { estimated } \\
\text { thickness }\end{array}$ & edge area \\
\hline $\mathrm{m}^{2} \mathrm{~g}^{-1}$ & sites $\mathrm{nm}^{-2}$ & $\mathrm{~nm}$ & $\%$ of total area \\
\hline Kaolinite KGa-1 & 11.6 & -0.3 & 40 to 70 & $20 \%$ to $30 \%$ \\
\hline Illite IMt-2 & 21.7 & -2.0 & 8 to 20 & $2 \%$ to $10 \%$ \\
\hline montmorillonite SWy-2 & 30.9 & -14.9 & 1 & $\mathrm{NA}$ \\
\hline polystyrene, amidine & 9.5 & +0.9 & 600 (diameter) & $\mathrm{NA}$ \\
\hline polystyrene, sulfate & 5.6 & -0.4 & 1,000 (diameter) & $\mathrm{NA}$ \\
\hline polystyrene, carboxylate & 5.6 & -13.7 & 1,010 (diameter) & $<1 \%$ \\
\hline
\end{tabular}

Table 1. Summary some size, shape, and charge properties of colloids tested. Surface area data for clays are from $\mathrm{N}_{2}$ BET measurements (Micromeritics, Norcross, GA). Surface area and charge data for polystyrene latex microspheres are from the manufacturer (Interfacial Dynamics Corp., Portland, OR). References for other mineral properties are noted in the text.

\section{Figure Captions}

Figure 1. Scanning electron micrographs of clays, (a) kaolinite, (b) illite, and (c) Namontmorillonite.

Figure 2. (a) Samples of kaolinite suspension taken at steady-state along the bubble-column (pH 5.7, $10^{-3} \mathrm{M} \mathrm{NaCl}$ ). Kaolinite particles were highly concentrated at the top (right side), and almost absent from the bottom of the column. (b) Measured and calculated relative 
concentration profiles of kaolinite particles within the bubble column $\left(10^{-3} \mathrm{M} \mathrm{NaCl}\right.$, various values of $\mathrm{pH})$.

Figure 3. The $\mathrm{pH}$ dependence of $K$ values for kaolinite clay. Kaolinite is highly surface active under acidic conditions. $K$ decreased rapidly when $\mathrm{pH}>6.3$, and reduced to zero when $\mathrm{pH}$ reached to 8. All experiments were conducted in $10^{-3} \mathrm{M} \mathrm{NaCl}$.

Figure 4. Ionic-strength dependence of $K$ values for kaolinite clay. Solution $\mathrm{pH}$ values were in the range of 5.4 to 5.8 .

Figure 5. Measured $K$ for illite at different $\mathrm{pH}$ values, particles were suspended in $10^{-3} \mathrm{M} \mathrm{NaCl}$.

Figure 6. Inverted concentration profiles of Na-montmorillonite at different $\mathrm{pH}$ values, showing surface exclusion of the Na-montmorillonite particles from the air-water interface. Similar results were obtained with bentonite clay. 